\title{
Extending State Transition Diagrams for the Specification of Human-Computer Interaction
}

\author{
ANTHONY I. WASSERMAN, MEMBER, IEEE
}

\begin{abstract}
User Software Engineering is a methodology for the specification and implementation of interactive information systems. An early step in the methodology is the creation of a formal executable description of the user interaction with the system, based on augmented state transition diagrams. This paper shows the derivation of the USE transition diagrams based on perceived shortcomings of the "pure" state transition diagram approach. In this way, the features of the USE specification notation are gradually presented and illustrated. The paper shows both the graphical notation and the textual equivalent of the notation, and briefly describes the automated tools that support direct execution of the specification.

This specification is easily encoded in a machine-processable form to create an executable form of the computer-human interaction.
\end{abstract}

Index Terms-Executable specifications, interactive information systems, rapid prototyping, software development methodology, transition diagrams, user interfaces, User Software Engineering.

\section{INTRODUCTION}

$\mathrm{A}$ $\mathrm{N}$ interactive system can be seen as having two components: the user interface to the system and the operations performed by the system. The user interface provides the user with a language for communicating with the system. The interface can take many forms, including multiple choice (menu selection), a command language, a database query language, or natural language-like input. In all cases, however, the normal action of the program is determined by user input, and the program may respond in a variety of ways, including results, requests for additional input, error messages, or assistance in the use of the system.

The user interface is often the principal determinant of system success, especially for those interactive systems where usage (or purchase) is discretionary. Yet for many systems used on alphanumeric terminals, design of the user interface is often an afterthought, with the design based on systemoriented, rather than user-oriented, concerns. ${ }^{1}$

The User Software Engineering project was undertaken in 1975 with the idea of combining concerns about user involvement in the design of interactive information systems with those of software engineering. The outcome of the effort is the creation of a methodology [1], [2] with a set of automated tools to support the methodology [3]-[7]. User participation is very important at the early stages of the meth-

Manuscript received February 8, 1984; revised February 20, 1985. This work was supported by corporate research grants from Nippon Electric Company and from Alcoa Foundation.

The author is with the Section of Medical Information Science, University of California, San Francisco, CA 94143.

${ }^{1}$ Interfaces for graphics-based systems and especially video games are based on the value of pictorial display and interaction. Therefore, more attention is given to the nature of the user interface. odology, where users (or user surrogates) can provide useful information to help the development process. For example, the initial analysis phase includes not only traditional data and activity modeling, but also identification of user characteristics, e.g., ability to type, intensity of anticipated usage, motivation and education of users, etc. Attention is also given to the environment in which the system will be used, so that the system can fit in with the user's work pattern, and can be tailored to any constraints on terminal types or transmission rates.

The second step of the USE methodology is external design, which involves design of the user interface(s) to the proposed system. The analysis step serves to identify major functions and required inputs and outputs, at least at a high level. The concern of external design is to determine how the user can request those functions and how the output will be displayed. Thus, instead of top-down or bottom-up design, the USE methodology uses an "outside-in" design.

The third step is the creation of an executable version of the user interface defined at the previous stage, so that the user and developer can jointly explore, both objectively and subjectively, the usability of the original design, and to make modifications as needed. This ability to rapidly create system prototypes, presenting the user view of the evolving system, is a key aspect of User Software Engineering. Clearly, there is iteration among the first three steps until one or more acceptable interfaces are found.

In the remainder of this paper, we describe the notation used to specify this user interface in the USE methodology. We are not concerned here with the methodological process of defining the user interface, with the succeeding steps of the methodology that lead to a finished system, or with evaluation of the user interface.

\section{USER INTERFACE Definition With TRANSITION Diagrams}

In searching for an appropriate notation for describing user interfaces to interactive systems, we established several requirements, including the following:

1) Formalism: The notation had to serve as a formal definition of the interface.

2) Completeness: The notation had to be self-contained, including user input, system output, and linkage to system operations (application code).

3) Comprehensibility: The notation had to be comprehensible both to system developers and to users (or their representatives). 
4) Flexibility: The notation had to accommodate a broad variety of dialog styles. In other words, the notation could not make assumptions about the nature of human-computer interaction, but had to give the designer of the dialog as wide a selection of possibilities as possible. This decision implies the need for a "low-level" approach to dialog specification.

5) Executability: The notation had to be directly executable to support prototyping, development, and testing of interactive information systems.

We observed that the interactive system and its actions are driven by raw or transformed user input. Accordingly, an effective specification technique for programming languages can be used effectively for specifying user interfaces. One can write down the grammar of the user input, and associate program actions with the successful recognition of "words" or "phases" in the grammar.

We decided to adapt transition diagrams for this purpose [8]. Transition diagrams have been used for a variety of language translators, including an early Cobol compiler [9], and are used as the formal specification of the MUMPS programming language [10]. (Transition diagrams have also been selected by others as an appropriate notation for specifying interactive programs $[11]-[15]$.)

A transition diagram is a network of nodes and directed arcs. Each arc may contain a token, corresponding to a character string in the primitive alphabet (such as ASCII), or the name of another diagram. If the path is blank, it will be traversed as the default case, i.e., if all other paths leaving a given node fail. Scanning of the diagram begins at a designated entry point and proceeds until reaching an exit node or a dead end (no successful match on the paths from a given node). An operation may be associated with any path; traversal of the path causes the associated operation to occur.

Intuitively, one can see that paths may contain arbitrary strings and that the state transitions can invoke arbitrary operations. The distinguished inputs then lead to different states from which other input symbols may cause yet additional actions.

We began by using a simple transition diagram model to design and build several small interactive systems. This model contained just three different symbols, as follows:

1) node-shown by a circle, representing a stable state awaiting some user input. Each node within a diagram has a unique name, and an output message may be displayed when a node is reached. One node is designated the starting node, designated by two concentric circles, and there is a single exit point.

2) arc-shown by an arrow, connecting nodes to one another. Each arc represents a state transition based on some input. The input is designated either by a string literal, such as "quit," or by the name of another diagram, enclosed within angle brackets, such as $<$ diag2 $>$. One arc emanating from each node may be left blank, in which case it becomes the default transition, and is taken only when the input fails to match that specified on any of the other arcs. We shall assume for the moment that there is no ambiguity concerning which arc to take for a given input at a given node.

3) operation-shown by a small square with an associated integer. An action may be associated with a transition to represent an operation that is to be performed whenever a spe-
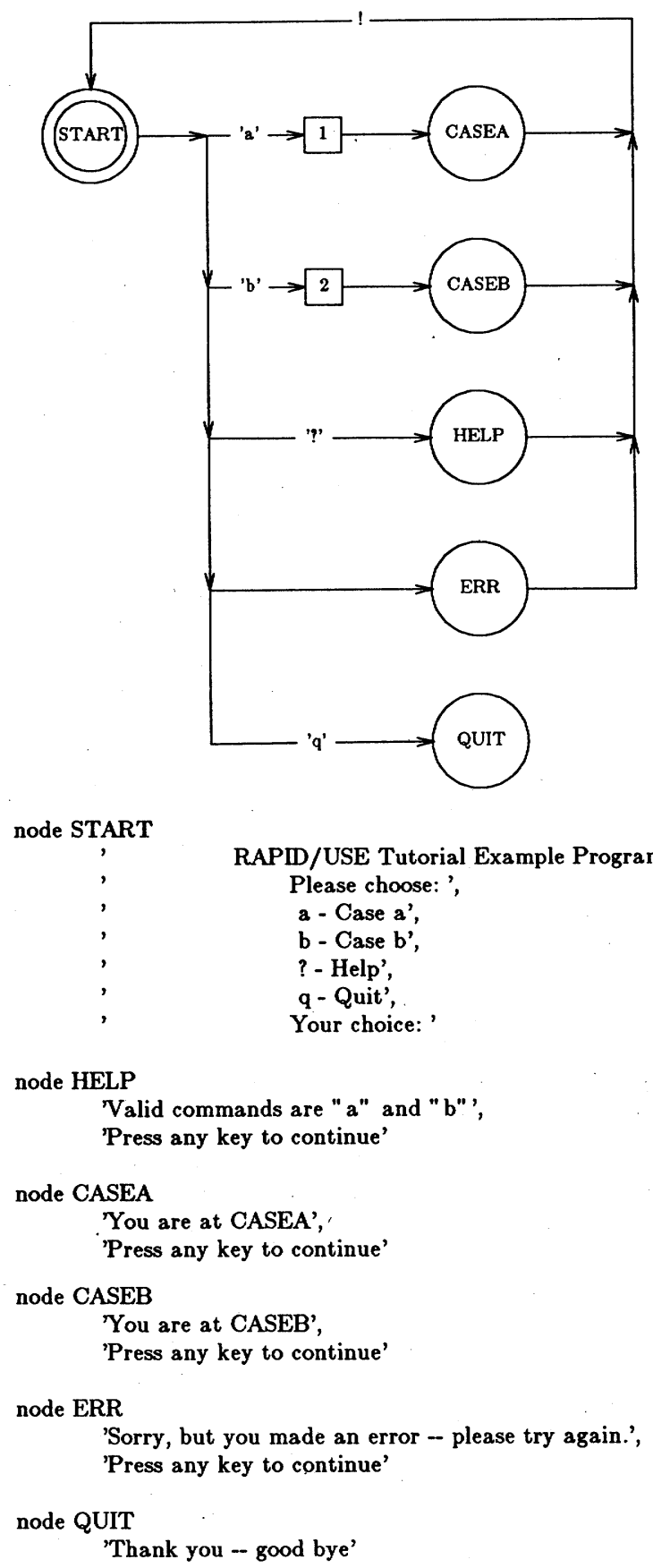

,

,

, a - Case a',

, b - Case b',

? - Help',

, q-Quit',

q- Quit',
Your choice:

node HELP

'Valid commands are " $a$ " and " $b$ "',

'Press any key to continue'

node CASEA

'You are at CASEA',

'Press any key to continue'

node CASEB

'You are at CASEB',

'Press any key to continue'

node ERR

'Sorry, but you made an error - please try again.',

'Press any key to continue'

node QUIT

'Thank you -- good bye'

Fig. 1. A USE transition diagram.

cific arc is traversed. The same action may be associated with more than one arc.

A very simple diagram is shown in Fig. 1. The diagram begins at START, where it waits for input. Input is a string terminated by a carriage return. There is a transition to one of five nodes based upon the input. The input of "a" causes action 1 to be performed during the transition to CASEA; the input of " $b$ " causes action 2 to be performed during the transition to CASEB, the input of "?" causes a transition to HELP, and the input of " $q$ " causes a transition to QUIT; anything other than "a", "b", "q", or "?" causes a transition to ERR.

The text beneath Fig. 1 shows the message displayed when a transition is made to that node. Thus, this figure is a very simple instance of a menu-selection dialog, with the START 
node used to present a menu, and the five other nodes representing two system commands, a quit command, a help command, and an error case.

We quickly recognized some significant shortcomings with this simple model, including the following.

1) Output Specification: It was important to specify formatting and layout of system output, rather than just describing the message itself.

2) Display of Input Information: It was not possible to include as part of an output specification any text that had been recognized as input from the user. Yet such display is a major component of many interactive programs.

3) Input Processing: Some interactive dialogs accept a line of user input, followed by a terminator, such as a carriage return, before performing an operation or making a transition, while others respond on a single key stroke or the recognition. of a specific key, such as a function key. The simple model we were using accepted only a variable length string, followed by a carriage return.

4) Diagram Complexity: Complex dialogs result in very complex diagrams, greatly reducing their comprehensibility and increasing the likelihood of errors in drawing or maintaining the diagrams.

5) Alternative Displays Based on Operations: The logical flow of an interactive program is often dependent upon the result of an operation, e.g., success or failure in a table lookup. Such a situation could not be represented with the simple transition diagram model.

6) Time Limits: It was not possible to make a transition after a fixed amount of time as needed to produce a remainder message.

We therefore sought to develop a transition diagram-like notation that would overcome these shortcomings. We also wanted to define a textual representation of these diagrams to facilitate their encoding for creating an executable version of the user interface. We shall henceforth refer to these diagrams as USE transition diagrams.

We now take up the approaches to these shortcomings that were introduced in USE transition diagrams.

\section{OUTPUT SPECIFICATION}

A node is used to display a message. In its simplest form, a message may simply be a text string, such as shown in Fig. 1. In practice, though, more power is needed for output specification.

\section{A. Cursor Control}

First, it is useful to take advantage of screen-oriented displays, specifying the exact or relative position on a screen at which a message should be placed. Thus, one might want to specify that the message begin on row 12 at column 25 , or that it begin two lines below the previously written message. We can specify the message as

\section{r12, c25, 'Good morning, fearless leader'}

It is often desirable to center a message on a line. Rather than counting spaces to find the correct starting point, the symbol "c " may be used to denote the center, so that the specification

\section{r12, c_'Good morning, fearless leader'}

would cause the message to be centered on line 12 .
We adopt the convention that the upper left hand corner of the screen is $(0,0)$ and the last row and column are each designated by "\$". Thus, a message to be written at the beginning of the last line could be addressed with $\mathrm{r} \$, \mathrm{c} 0$. A relative movement is designated with " + " and "-," so that one could write

$$
\mathrm{r}+2, \mathrm{c} 25, \text {,Your request...', }
$$

In many cases, a message may contain an entire screen full of information, so that the message could contain an arbitrary mixture of text and positioning directives. One could write, then,

$$
\begin{aligned}
& \text { cs, r5, c5, 'Please choose one of the following:', } \\
& \mathrm{r}+2, \mathrm{c} 10, \text {,1) deposit', } \\
& \mathrm{r}+2, \mathrm{c10}, \text { '2) withdraw', } \\
& r+2, \text { c10, '3) help', } \\
& \mathrm{r}+2, \mathrm{c10}, \text { '4) quit', } \\
& \text { r\$, rv, 'Your choice...', sv }
\end{aligned}
$$

to specify a screen filled with text.

Note that screen-oriented directives are used in this example.

$\begin{array}{ll}\text { cs } & \text { clear screen and go to home position (r0, c0) } \\ \text { rv } & \text { use reverse video (if available) } \\ \text { sv } & \text { return to standard video }\end{array}$

Additional screen-oriented directives provide needed cursor control. In all cases, these directives may be included in an output specification.
$\mathrm{hm} \quad$ go to home position $(0,0)$
nl start a new line $(\mathrm{r}+1, \mathrm{c} 0)$,
cl clear to end of line on current row
ce clear to end of screen from current line
dc delete the character at the current position, then shift subsequent characters on line to left
d1 delete entire line (current row) and scroll lower lines (if any) upward
is_'text' insert the text beginning at the current position
il open a blank line at the current position and move lower lines down

\section{B. Reuse of Messages}

Second, we may want to reuse a message. Common examples of this situation are error messages, screen headings, and online assistance. The structure of a message is identical to that of a node specification, so that a message may include cursor movement information, text strings, and variable names. We define a message name, then refer to it by that name whenever we want to display that message. Conceptually, we would define

message prompt

$$
\text { r\$, rv, 'Your choice...', sv }
$$

and we then write

$$
\begin{aligned}
& \text { cs, r5, c5, 'Please choose one of the following:', } \\
& r+2, c 10, ' 1) \text { deposit', } \\
& r+2, c 10, \text { '2) withdraw', } \\
& \text { r }+2, c 10, \text { '3) help', } \\
& \text { r }+2, c 10, \text { '4) quit', } \\
& \text { prompt }
\end{aligned}
$$


The appearance of the message name "prompt" causes the substitution of the text associated with the message definition of "prompt."

\section{Tab Settings}

The layout of the previous example is dependent upon the alignment of four lines of text, requiring the inclusion of the directive "c10" in four different places. Changing the placement of these lines requires changing four occurrences of the column directive in this message, and potentially other dependent column directives in other messages that might be concurrently displayed. Flexibility and the ability to modify layouts rapidly suggests the need to associate tabs with column settings.

We introduce the tab definition and allow a column to be associated with a specific tab declaration, as follows.

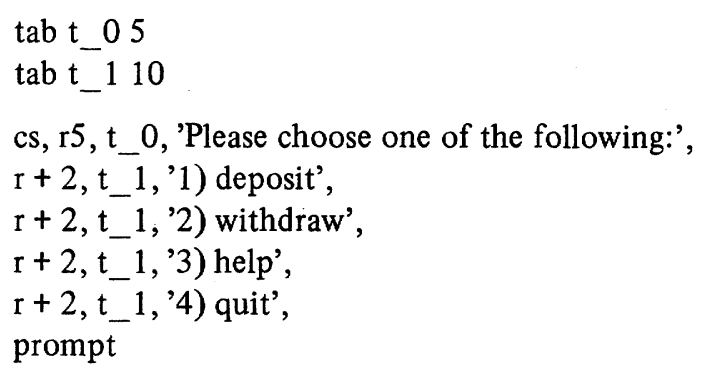

In this way, the alignment may be changed with only one change to the definition, namely the column associated with the tab definition.

\section{Partitioned Screens}

Another problem in controlling a display is to be able to partition the screen into two or more parts and to move freely between them. For example, we may wish to use the bottom two lines of the screen for error messages, regardless of what has been placed on the remainder of the screen. In that case, we would like to "mark" a position on the screen, move to another position, and then return to the marked position.

The directives "mark" and "tomark" provide part of this capability. The upper and lower case letters may be used as names of marks and then referenced. Named marks may be included as part of the output specification. The nodes

node one

$$
\mathrm{r}+1 \text {, 'Your reply: ', mark_A }
$$

node error

$$
\text { tomark_A, cl, }
$$
r\$, mark_E, rv, 'Please type a number from 1 to 5', sv, tomark_A

allow an erroneous reply to be cleared, and an error message to be displayed on the bottom line (in reverse video), after which the cursor returns to the point at which the reply is wanted. Note also that mark_E is set to the beginning of the error line so that some other node could be defined

$$
\begin{aligned}
& \text { node clean_err } \\
& \text { mark_B, tomark_E, cl, tomark_B }
\end{aligned}
$$

to remove the error message at a subsequent point. The removal should not be done in node one, since it would disappear from the screen before the user had a chance to read it.
This screen partitioning is a first step toward the multiple window interfaces employed in systems such as Smalltalk-80 and Interlisp [16] , [17]. We return to this point later.

\section{VARIAbles in Diagram Specification}

Output messages are often dependent upon previous input or upon computed results. Data entry systems must display and/or reformat information input by a user, as well as passing that data to operations. Programs involving multiple screens typically redisplay data given on one screen on a subsequent display. For example, a bank teller program may obtain account information, and then display the account holder's name or account number later in the interactive dialog. Thus, the limitation of fixed text in the output specification is inadequate, and we must introduce variables. We adopt the convention that a variable may be assigned the string received on a specific input, or be assigned a value as the result of an action. The variable name is shown on one or more arcs in a diagram. When such an arc is traversed, the input string is assigned to that variable.

Thus, the appearance of a variable name on an arc emanating from a node means that the input is assigned to that variable.

If there were a message such as

cs, r10, c10, 'Please type your name:'

the resulting input could be assigned to a variable called "name," and could subsequently be displayed as follows:

$$
\text { r0, c40, 'User--', name }
$$

within a node or another message.

As with variables in programming languages, we wish to define constraints on their values. We define four kinds of constraints:

1) data type: string, integer, float, scalar, date, time

2) string length: minimum and maximum length (both optional)

3) range of values: lexicographic or numeric ranges, depending on type (both optional)

4) display format: used as default format for displaying values of the variable.

Thus, all of the following are variable definitions:

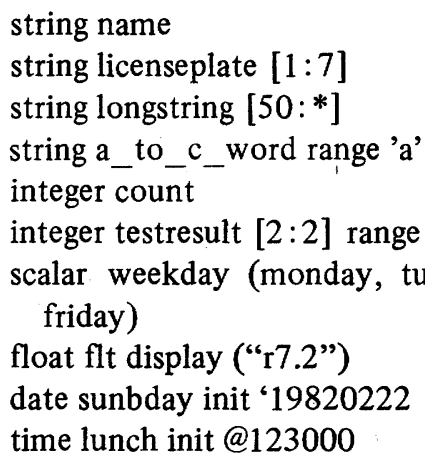

The numbers in square brackets delimit the length of the input in characters. The range constraint specifies a restriction on the value of the variable, where the range for "integer" is given by integer values and the range for "string" is given by the ASCII collating sequence. An asterisk "*" may be used to denote the absence of a fixed limit. The syntax for the 
display format is similar to that used for the printf function in the Unix ${ }^{\mathrm{TM}}$ standard $\mathrm{I} / \mathrm{O}$ library, and has been extended to support the display of scalars, month (numeric and text), day, year, weekday, hours, minutes, and seconds, as well as left, right, and center adjustment of displayed variables.

Variables may be assigned by appearing on a transition, so that user input meeting the constraint(s), if any, causes the transition and makes the assignment. In Fig. 2, the transition from step 1 to step 2 causes the variable "restname" to be assigned the user input string.

Once a variable has been assigned a value, it may subsequently be used in messages and in actions. As with variables in other programming notations, it may also be assigned a new value.

\section{InPUt Processing}

The basic transition diagram model assumes that the input to determine transitions along an arc is a variable length string terminated by a carriage return. Also, there must be some input to cause a transition. All of these assumptions are unnecessarily restrictive, in addition to being unrealistic for the practical design of interactive systems. Therefore, extensions to the transition diagram model are needed for these cases.

First, though, we observe that the input character set must be extended beyond the typical set of 95 ASCII printable graphics, to accommodate control characters, function keys, and other inputs that may be received from a modern terminal keyboard. We use the following symbols to represent the extended input character set.

$\begin{array}{ll}\text { esc } & \text { escape } \\ \text { cr } & \text { carriage return (default) } \\ \text { bs } & \text { backspace } \\ \text { If } & \text { line feed } \\ \text { del } & \text { delete } \\ \text { tab } & \text { tab character (ctl_i) } \\ \text { ctl__A-Za-Z }\} & \text { any control character, such as ctl_D, for any } \\ \text { f }\{0-9\} & \text { letter } \\ \uparrow & \text { function keys f0 through f9 } \\ \downarrow & \text { up arrow } \\ \leftarrow & \text { down arrow } \\ \overrightarrow{\text { home }} & \text { left arrow } \\ \text { right arrow }\end{array}$

Thus, in the same way that one can write a string literal on an arc, one can write any of these symbols, treating them as "reversed words" and prohibiting the use of variables with these names.

\section{A. Buffered versus Unbuffered Input}

As noted, the assumption of inputs terminated by carriage returns is very restrictive, and fails to represent many of the most common uses of interactive systems. While we use that assumption as a default, we need mechanisms for overriding that case.

First, a single keystroke may be used to determine the appropriate branch, without a terminating character. This ap-

${ }^{\text {TM }}$ Unix is a trademark of AT\&T Bell Laboratories.

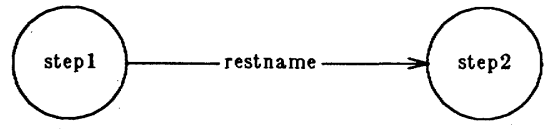

Fig. 2. Assignment to a variable.

proach is commonly found in multiple-choice (menu selection) applications, not only with keyboard input, but also with touch screens and mouse input. Indeed, single keystroke (or equivalent) processing is a key aspect of many highly interactive systems. Rather than buffering the input, it must be processed immediately.

Thus, the two forms of input processing must be distinguished, which cannot be done with the simple model of transition diagrams. We introduce the "!" symbol to denote this immediate transition, which we term a single key transition. The appearance of the "!" followed by a character on an arc means that a single "character"' input is used to cause a transition. Fig. 3 gives an example of this case.

\section{B. Specific Character Recognition}

A similar problem arises when it is desired to make a transition based on the recognition of a particular character, whenever it appears in the user input. This is termed a special key transition, and is shown by preceding the key with an ampersand ("\&") on the arc. The ampersand indicates the immediate transition, without waiting for a terminator, rather than accepting input until a terminator is received.

Note that "single key" and "special key" transitions are difficult. In a command-oriented system, it may be possible to interrupt a command or any other input by typing a specific key, such as control-C or "?". It is only the "special key" concept that permits this interruption, unless all input is handled as "single key."

We have also considered the case in which a specific key, such as an escape or a function key, can be globally used to terminate a diagram and perhaps return to a specific node in a specific diagram. From a notational standpoint, it is necessary to have an explicit arc from every node where such an input could be received. (It is straightforward to implement this idea in software, though.) Without such an explicit arc, a diagram (or, as we shall see, a set of diagrams) may have unconstrained flow of control (the goto problem); furthermore, such branching in a set of diagrams raises traditional programming language questions about the scope of variables.

\section{Truncating Input String Length}

The default case in transition diagrams is to accept a string of any length until a carriage return is received. We wish first to consider strings of fixed length (frequently length 1 ), and also strings terminated by something other than a carriage return. Consider the very common case of asking a user for a "yes" or "no" response. The decision can normally be made by examining the first character of the user input, so that a variety of different response can be handled similarly. Even in this simple case, the truncation greatly simplifies the problem of decoding input, which might be any of "N," " $n$," "NO," "No," or "no," excluding numerous possibilities from languages other than English. Note that the length option is different from the single_key option in that the length trun- 


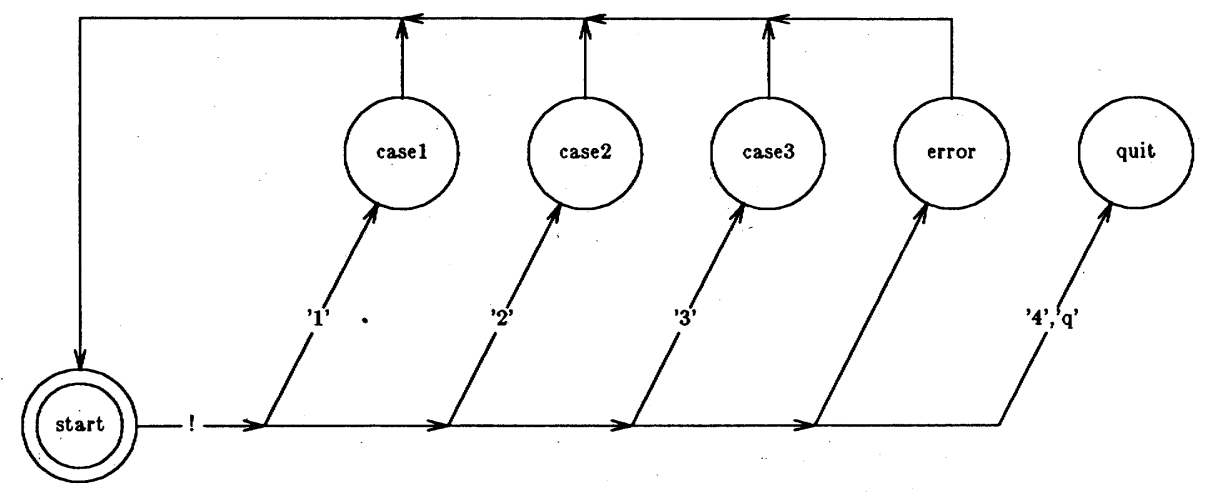

Fig. 3. Transition diagram illustrating immediate transition.

cation is only applied after the input termination character (normally the carriage return) is received.

\section{Nonstandard Terminators}

The carriage return default may be overridden with the until option. In the vi text editor for the Unix operating system, for example, insertion mode is terminated with the "escape" character. In other applications where the input may extend over several lines, the carriage return must be treated as a normal input character, with the terminator switched to a different character. Thus, one might wish to describe that all input received prior to an escape character will be assigned to a variable named inputstr.

The until feature may be combined with the length feature, so that an input string may be truncated to a fixed length regardless of the terminator. Fig. 4 illustrates the notation used in the USE transition diagram for nonstandard terminators and/or fixed length strings. This information precedes the input string(s) and is denoted by the slash symbol "c/". A list of zero or more alternative terminators is given to the left of the " $/$ " and the length, if fixed, is given to the right. The fragment of the diagram shown in Fig. 4 indicates that input is read until an escape or a tab character is received, and then truncated to eight characters. The resulting string is assigned to variable instring. These extensions to input processing, the extended character set, single key input, special key transitions, alternate terminators, and input string truncation, allow the description of a much greater set of interactive dialogs than was previously possible.

\section{Diagram Decomposition}

The added expressiveness given by the output specification notation, the inclusion of variables, and the extensions to input processing made it possible to describe most interactive systems using alphanumeric display terminals. The USE transition diagrams could be used with a means for specifying the actions, and thereby serve as a specification method.

One important aspect of specifications, though, is comprehensibility. We found that it was very easy to create large complex diagrams that could not easily be written or understood. Diagram complexity arose initially from the desire to provide error handling and help facilities in the interactive dialog. By following our own guidelines for designing inter-

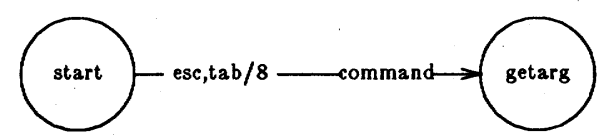

Fig. 4. Nonstandard terminators in USE transition diagrams.

active systems [18], we were led to provide a help arc and an error arc from each node where user input could occur (virtually all). Accordingly, the number of nodes and arcs proliferated. The situation was even worse if one wanted to provide a different message on the second occurrence of an error than on the first.

At first, we simply adopted the strategy of drawing a set of diagrams with no error handling and no interactive help facilities so that the diagram presented the "normal" flow of dialog. Yet this was also inadequate as we attempted to model increasingly complex dialogs and systems.

\section{A. Subconversations}

The solution taken was to introduce a hierarchy of diagrams, so that any diagram could "invoke" another diagram. A connected set of nodes could be named, and could be "called" from another diagram. This idea of a "subconversation" was useful because it also modeled a commonly occurring situation in interactive systems: a set of "transaction types." Accordingly, a fourth symbol was added to the node, arc, and action:

4) subconversation-shown by a rectangular box, with an associated diagram name. When an arc enters a subconversation box, traversal of that diagram is suspended, and control is transferred to the starting node of the diagram named in the subconversation box. The new diagram is then traversed until its exit point is reached, at which point control returns to the "calling" diagram, and the subconversation box is exited.

A simple example of this situation is shown by the diagram structure of Fig. 5, where there are subconversations for "deposit," "withdraw," and "balance.." The capability for any node to have multiple exits is retained in the subconversation by permitting a diagram to return a value to the invoking diagram so that the branch upon exit from the subconversation may be determined by the return value. The returned value is denoted by a nonnegative integer preceded by the "\#" symbol in the invoked diagram. If more than one arc emanates from a subconversation box, the associated return value used to determine the branch can appear on each arc. We adopt 


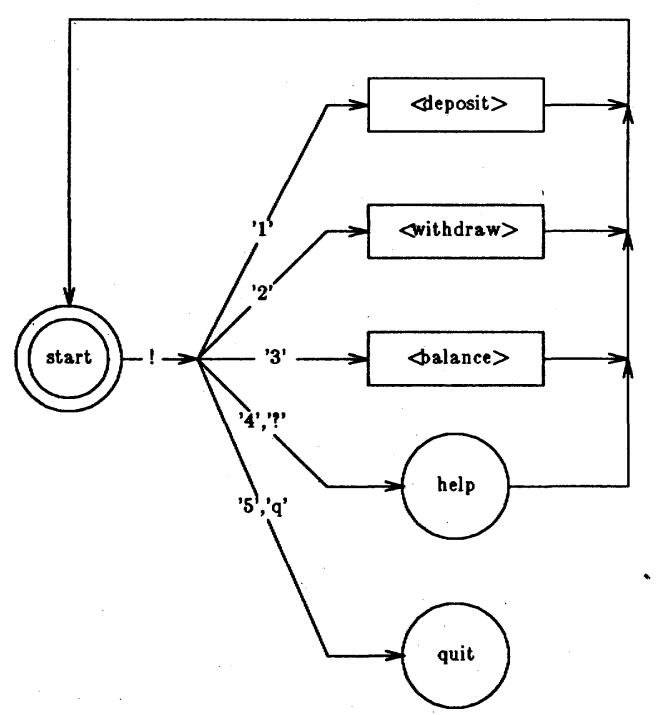

Fig. 5. The use of subconversations for transactions.

the convention that such values may only be returned to the immediate caller, i.e., one level of invocation. This use is shown in Fig. 6.

\section{B. Transitions Described by Diagrams}

Until now, we have assumed that all inputs are described either by a string literal (a directly named sequence of characters) or by an input string that is immediately assigned to a variable. In some cases, though, it is useful to use a diagram to describe the syntax that causes a transition. Rather than using a string literal or a variable name on an arc, one can use the name of a diagram. That diagram, and any additional levels invoked from that diagram, must be successfully traversed to cause the given arc to be traversed. This situation is shown in Fig. 7; in Fig. 7(a), the diagram name $\langle x$ toz $>$ is associated with an arc connecting nodes "one" and "two," while Fig. 7(b) shows the diagram xtoz. Note that $<$ atob $>$ is another possibility for the traversal from "one" to "two;" which can also be tried.

This use of subconversations allows recognition of a string composed of several components, possibly of indeterminate length, without overly complicating the higher level diagram. As before, the called diagram could be inserted into the higher level diagram, without altering the effect. In this case, though, it becomes possible to hide the structure of the recognized string. This construct is also useful for allowing several different strings to cause the same transition, which is frequently needed for dialogs supporting novice and expert interfaces, for example.

\section{Decomposing Messages and Actions}

Another important option is the ability to decompose messages and/or actions. Suppose that we want to perform action 2 on one arc, actions 2 and 3 in response to another input, and actions 1 and 2 in response to yet another input. To do so, one must create an intermediate node to permit the actions to be specified independently. Yet we do not want to require additional input to cause the second action to occur. Simi-

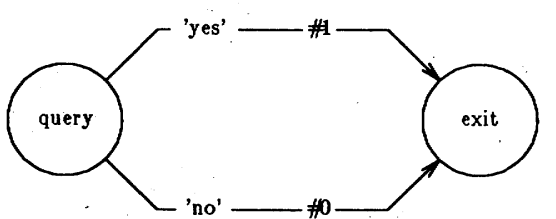

Fig. 6. Returning values from subconversations.

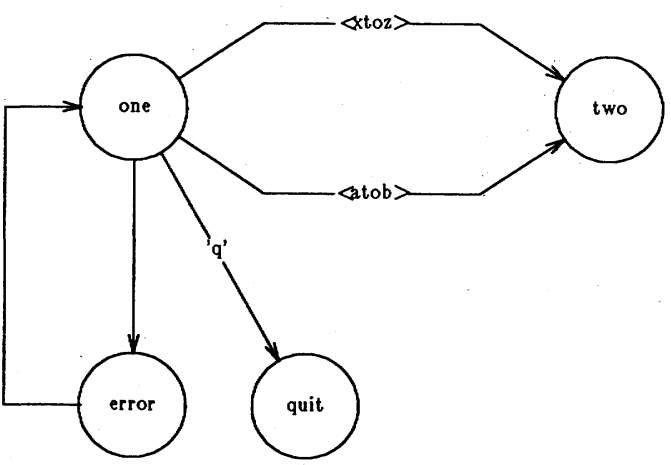

(a)

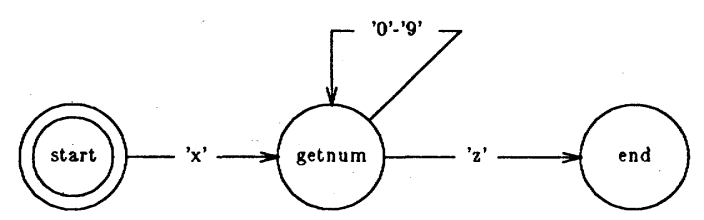

(b)

Fig. 7. (a) Using diagram names for arc traversal. (b) Use of a subconversation for string recognition.

larly, one may wish to display two separate messages in response to a single input without waiting for additional input from the user.

In both of these cases, one must make an automatic transition from one node to another. This case, called the skip case, is denoted by a "+" on an arc; of course, no string, return value, or diagram name can appear on such an arc.

\section{User Interfaces as a Hierarchy of USE Transition Diagrams}

The use of subconversations is both an aid to diagram decomposition and a notational convenience. There are, however, several distinct advantages to their use.

1) Any diagram may be referenced from other diagrams in a dialog and thereby reused; this supports the creation of libraries of diagrams that can be integrated into systems.

2) A higher level diagram can often be designed without making decisions about the actual input text; this approach allows decisions about the dialog to be deferred and isolated, and therefore changed easily.

3) Subconversations help to break up a diagram that has a large number of nodes; to aid comprehension, diagrams should contain fewer than 10 nodes.

4) Subconversations help to illustrate the structure of a dialog; many dialogs are naturally hierarchical and the subconversation mechanism allows this hierarchy to be shown in the diagrams. 


\section{Semantic Aspects of USE Transition Diagrams}

Until now, transitions between nodes in diagrams have been driven by the syntax of the user input. The extensions have either been structural, e.g., subconversations, or directed to finer input control, e.g., unbuffered input. Even the introduction of variables into diagrams did not alter this situation. However, it now becomes necessary to introduce semantic dependencies into USE transition diagrams.

\section{A. Returning Values fróm Actions}

We previously noted that the direction of a dialog is often dependent upon the result of an action. For example, in a banking system, the user (a teller) would be asked to input a customer account number. A subsequent action would be to look up this account number in the bank's customer account database. The next message presented to the teller would depend on the success of the search.

To achieve this effect, it is necessary to associate a return value with the action, and then to branch on that value. This is easily accomplished in our notation by indicating one or more arcs emerging from an action box, with arcs labeled with alternate return values, following the same approach used for subconversations. This situation is shown in Fig. 8. One path, labeled 1 , leads to node found, while the other path, labeled 2 , leads to node notfound. Note that the continuation from an action may be either unconditional, as we have previously seen, or conditional based on a return value. In the unconditional case, there may be a returned value, but it will not affect the transition following the action.

\section{B. Time Limits}

In modeling interactive systems with state transition diagrams, we found no convenient way to express time, since transitions are linked to user input. In practice, though, one often expects user input within a fixed amount of time, with an unexpected delay indicating a problem. Thus, it is desirable to be able to effect a transition on the expiration of a predefined time limit. In this way, it is possible to branch to another node, from which a reminder or help message can be displayed.

We thus introduce the alarm transition, and denote that transition by writing the time limit on the appropriate arc, e.g., $30^{\prime \prime}$. The alarm transition is made if no input is received from the user before the time limit expires.

\section{AN EXTENDED EXAMPLE}

To this point, all of the examples of the USE transition diagram specification method have dealt with "toy" examples, intended to denote the overall style and scope of the specification method. In this section, we show a small part of a much larger example, a data dictionary system to support a variant of Structured'Systems Analysis [19]. This example not only shows a broad range of the features of the USE specification method for interactive systems, but represents a running application system in everyday use. Because of space limitations, though, only three of the 31 diagrams representing the system are shown, and the display is limited to the diagrams themselves (with the associated text and action calls),

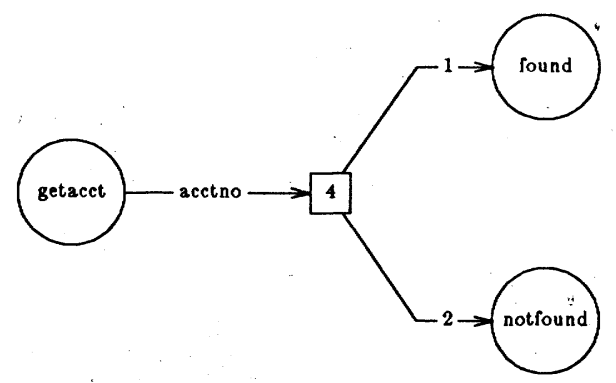

Fig. 8. Branch determined by value returned from action rules.

omitting the description of the actions on which they operate.

The data dictionary is represented by a set of relations, specified in the Troll/USE data definition and manipulation language, and shown in Fig. 9 [5], [20].

The first diagram, the main diagram for the data dictionary system, is shown in Fig. 10(a). Three features are worth noting in this diagram.

1) The action box numbered " 2 " is a call to the database script "startup." If this action succeeds (return "ok"), then control flows to node "start;" otherwise, control flows to "nodb" and " $x$ " and the program terminates.

2) The center of activity is node "select," which provides a menu-like interface to the user, providing the user with the ability to enter the subconversations "add," "modify," "delete," and "query." The "select" node also provides for terminating the program, asking for help, and handling unexpected input (the "error" node).

3) The structure found in this diagram is generic for transaction-oriented interactive programs. Many interactive information systems exhibit the same structure, and this diagram can be easily modified to suit other applications. (Note the similarity to Fig. 5.)

The subconversation "add" is shown in Fig. 10(b), and has much the same structure as does the top-level diagram, asking the user to specify the type of entry to be placed in the data dictionary. (Clearly, this dialog has been designed to minimize typing and to present a screen-oriented interface to the user.)

Note that the subconversations "add element" through "add_message" all return the value 0 if the item is successfully inserted in the data dictionary. This return value is used to direct control flow to node "inserted," which displays the message "successful insertion" before prompting the user for another entry.

The "add" subconversation then invokes "add_process" when the user wishes to define a new process and store information about that process in the data dictionary. Several aspects of the diagram shown in Fig. 10(c) are worth noting.

1) Three different kinds of actions are performed in this diagram. The actions "checkpro," "inspro," and "delparams" operate on the database shown in Fig. 9; as indicated by the use of the "call" to the actions. The action "CallEdit" is to an executable program, to be written in a high level programming language; it is, in fact, a call to the vi editor for a file to hold the process description for the given process. Finally, there is a case statement, denoted by the "cs" action box, which controls branching after the "checkpro" action is per- 


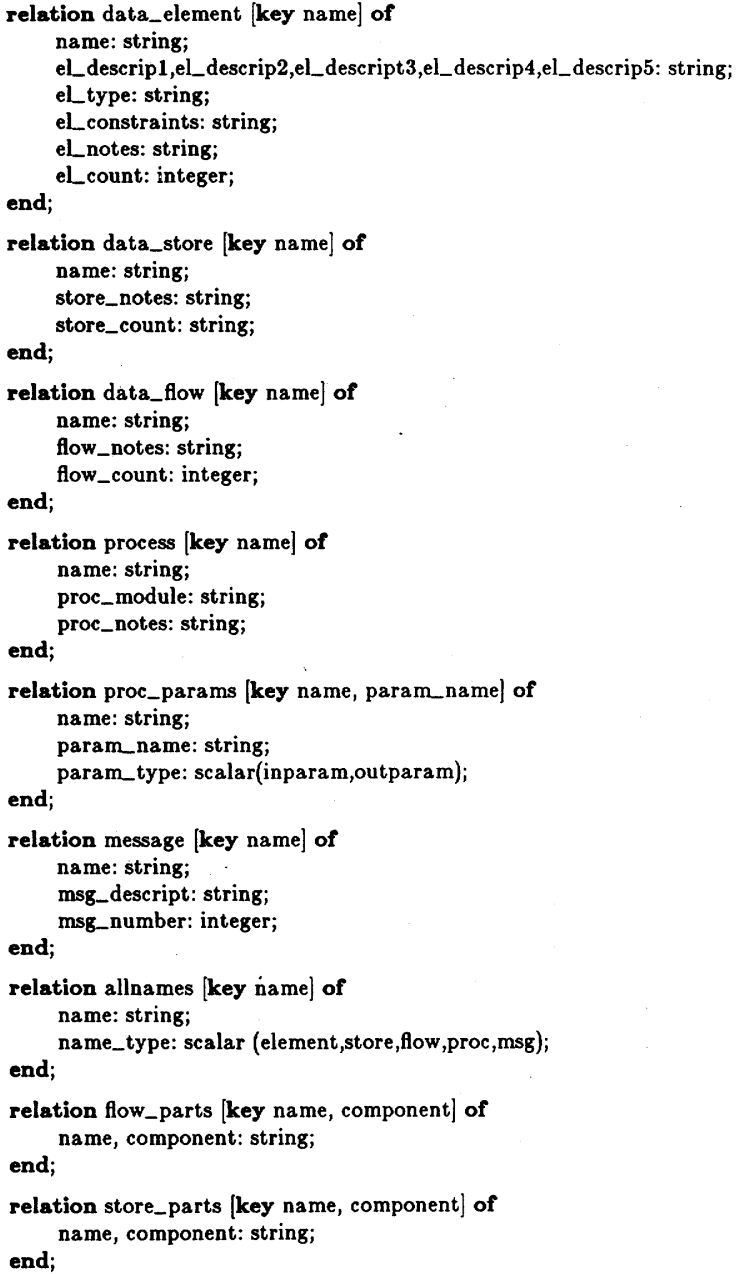

Fig. 9. Relational database schema for data dictionary.

formed. The USE transition diagram notation does not allow two consecutive action boxes, so it is necessary to interject the dummy node "hack1" between the call to "checkpro" and the case statement.

2) Three different values may be returned to "add," representing successful insertion of the information (0), an error in insertion (1), or user cancellation of the insertion (2). Referring back to Fig. 10(b), we note that the "add" diagram does not presently distinguish between the latter two cases.

3) The arc "skip" feature, denoted by "t", is used several times, when actions and/or displays are performed without intervening user input. Without the skip feature, it would be necessary to combine information that is logically separate or to require user input in the interim.

4) The message "main.lastline" is used, allowing the dialog designer to define some standard message formats in the main diagram and then use them throughout the dialog. This feature improves consistency of the interface design for the user.

\section{Executable Specifications}

As described in the introduction, a critical step in applying the User Software Engineering methodology is to create an executable version of the user interface. To this end, we have designed and built a system called RAPID/USE, which consists of two components: the transition diagram intepreter (TDI) and the action linker [3], [21]. The TDI was designed to accept an encoding of the USE transition diagrams that resembles the diagrams and messages as much as possible. ${ }^{2}$ This encoding, called a dialog description or a script, is transformed into tables by TDI. In this way, one can draw the diagrams and quickly transform them to an executable form. The encoding may be achieved either by editing the textual representation of the diagrams, or by using a graphical tool, the transition diagram editor [22], to draw the USE transition diagrams interactively, and to have the TDI dialog description generated automatically.

Input to TDI consists of one or more diagram descriptions, each representing a transition diagram. Each diagram may have five types of statements.

1) Diagram name statement-identifies the diagram, its entry node and exit node.

2) Variable definition statements-permit the use of names to describe strings of alphanumeric or numeric characters, along with range constraints on their values.

3) Message definition statements-permit the use of names to describe messages that are to be called from multiple points in the diagram.

4) Node definition statements-define the node names for the diagram, along with the associated messages and screen control for each node.

5) Arc statements-describe the structure of the diagram and its transition conditions.

The Action Linker part of RAPID/USE allows programmed actions to be associated with the transitions. Routines may be written in C, Fortran 77, or Pascal. (Linkage to other languages can also be provided.)

The syntax for the nodes is virtually identical to that shown in Fig. 10(a)-(c). The description for the arcs is given by the name of the starting node, followed by all of the possible branches and actions emanating from that node. Fig. 11 shows the TDI text for the diagram shown in Fig. 10(a). An inspection of this text will show that it is a direct encoding of the diagram itself. This encoding is done automatically by the transition diagram editor, and may also be done manually by someone without access to the graphical editor. Thus, RAPID/USE is used both for building and validating user interfaces (TDI) and for building functioning systems (TDI + Action Linker).

\section{Further Extensions to Dialog Specifications}

The USE transition diagrams, as described so far, cover a very broad range of the interactive dialogs that are suitable for user interfaces on "intelligent" alphanumeric display terminals. Furthermore, both the diagrams and the accompanying TDI notation are independent of the physical characteristics of any specific terminal. Thus, these diagrams may serve as a general descriptive technique for interactive systems.

With recent advances in computer terminals and worksta-

\footnotetext{
${ }^{2}$ The present distribution of RAPID/USE supports all of the transition diagram features described in Sections III-VII except for some restrictions on transitions described by diagrams (Section VI-B).
} 
main

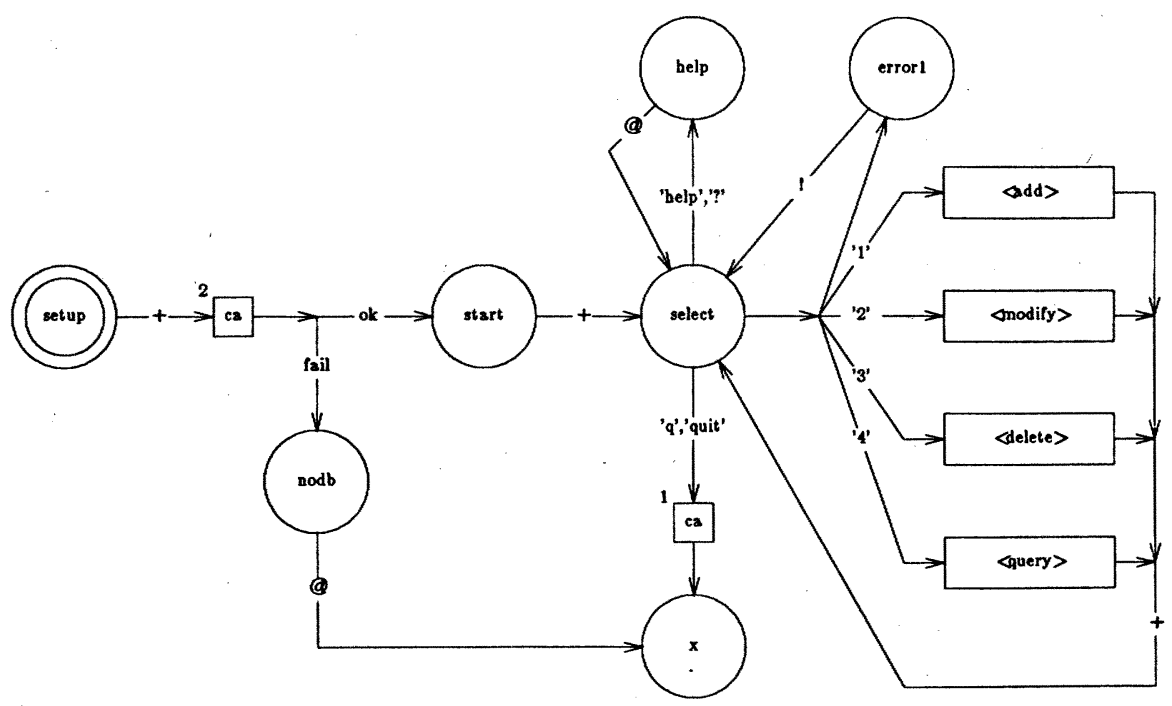

Actions

$\begin{array}{ll}1 & \text { call shutdown } \\ 2 & \text { call startup }\end{array}$

diagram main entry setup exit $x$

database 'usedddb'

library '../scripts'

tab t_o 15

tab t_1 20

message header

cs,r2,c0,c_'USE Data Dictionary'

message lastline

r\$,c0,'Hit any character to continue.'

node setup

node select

tomark_A,ce,r+3,t_0,'Please choose ',

$r+2, t \_1, ' 1$ : Add a dictionary entry.',

$r+2, t, 1,2$ : Modify a dictionary entry.',

$r+2, t \_1,3$ : Delete a dictionary entry.',

$r+2, t_{-} 1,{ }^{\prime}$ : Query data dictionary',

$r+2, t_{-} 1$,'help: Information on use of program',

$r+2, t+1$,'quit: Exit USE/Data Dictionary',

$r+2, t \_0, ' Y o u r$ choice:'

node help

cs, $\mathrm{r} \$-3, \mathrm{c0}$,'For more information about a command, enter',

r\$-2,c0,'the command number, press return and then type " help" or "?" ',

r\$, c0,'Hit any key to continue'

node nodb

cs,r\$,c0,'Could not open database directory'

node start

header,mark_A

node $x$

cs

node error 1

r\$-1,c0,rv, bell,'Please type a number from 1 to 4.',sv,

lastline

(a)

Fig. 10. (a) Top-level USE transition diagram of data dictionary system. 
add

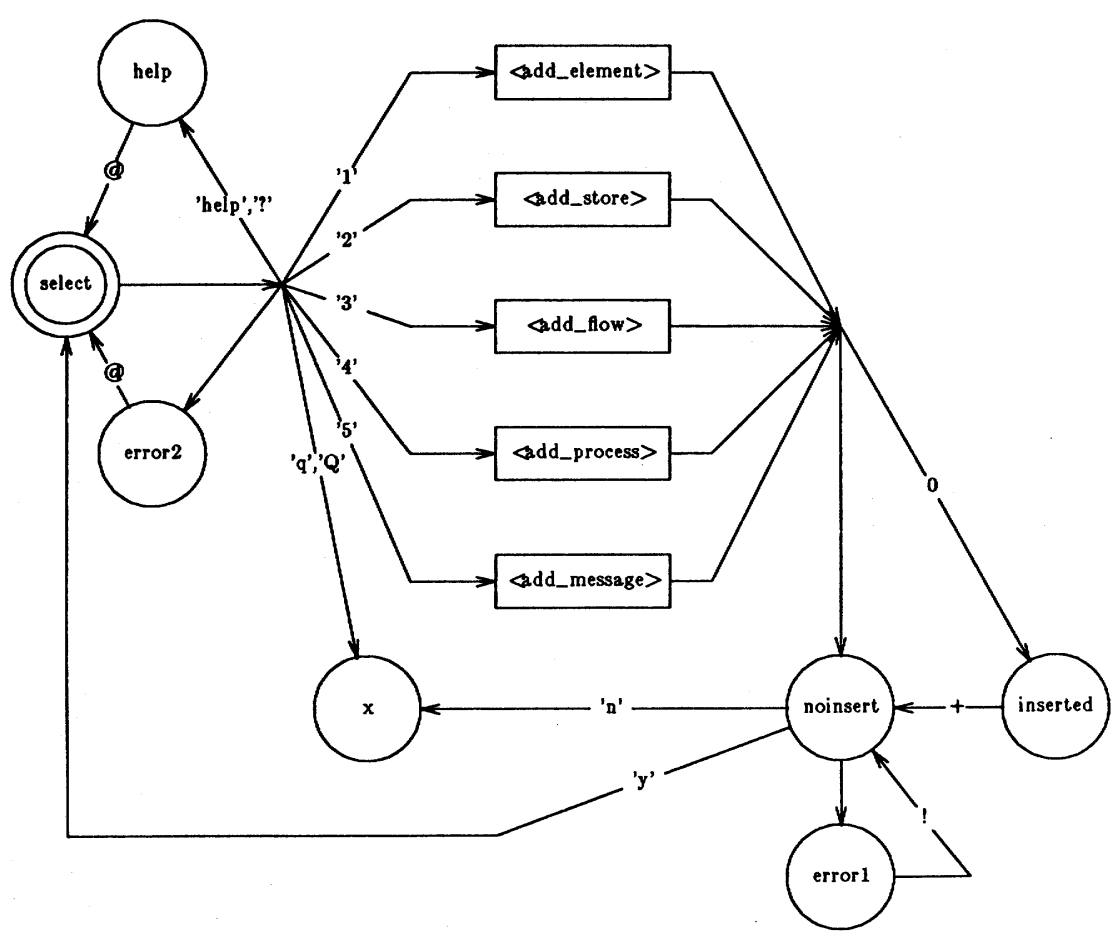

diagram add entry select exit $\mathbf{x}$

tab t_o 10

tab t_1 15

node error 1

r\$,c0,rv,'Please type "y" or " n" ',sv,tomark_E

node select

main.header,

r4,c0,c_'Add entry to dictionary',

$r+2, t \_0$,'Please select type of data entry: ',

$r+1, t_{-} 1,1$ : data element',

$\mathbf{r}+1, \mathbf{t} \_1,2$ : data store',

$\mathrm{r}+1, \mathrm{t}_{-} 1,{ }^{\prime} 3$ : data flow',

$\mathrm{r}+1, \mathbf{t} \_$, ', 4 : process',

$r+1, t_{-} 1, ' 5$ : message',

$r+2, t \_0$, 'Your choice (1-5): '

node help

cs,r0,c0,c_'USE/Data Dictionary',

r12,c0,c_'Add new entry',

main.lastline

node $x$

node inserted

r\$-4,c0,'Successful insertion'

node error2

r\$-1,cs,rv,bell,'Please type a number from " 1 " to " 5 " ',sv,

r\$, c0,'Hit any character to continue.'

node noinsert

$r+2, c 0$, 'Another entry $(\mathrm{y} / \mathrm{n})$ ?', , mark_E

(b)

Fig. 10. (Continued.) (b) The "add" subconversion of data dictionary system. 
add_process

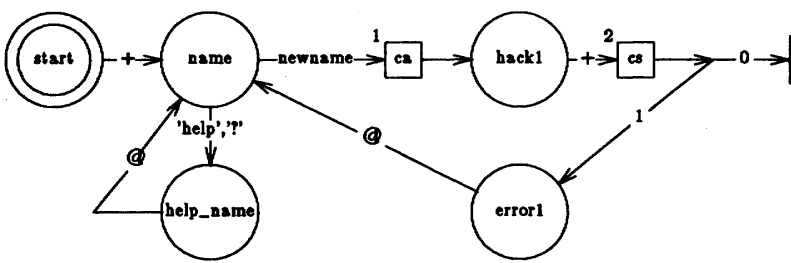

Actions

$$
\begin{aligned}
& \text { call checkpro(newname-> dups) } \\
& \text { case dups } \\
& \text { call inspro(newname,newnotes) } \\
& \text { call delparams(newname) } \\
& \text { do CallEdit("vi \%s.pdl", newname) }
\end{aligned}
$$

diagram add_process entry start exit $\mathbf{x}$

digit dups

alpha newname

alpha newnotes

node start
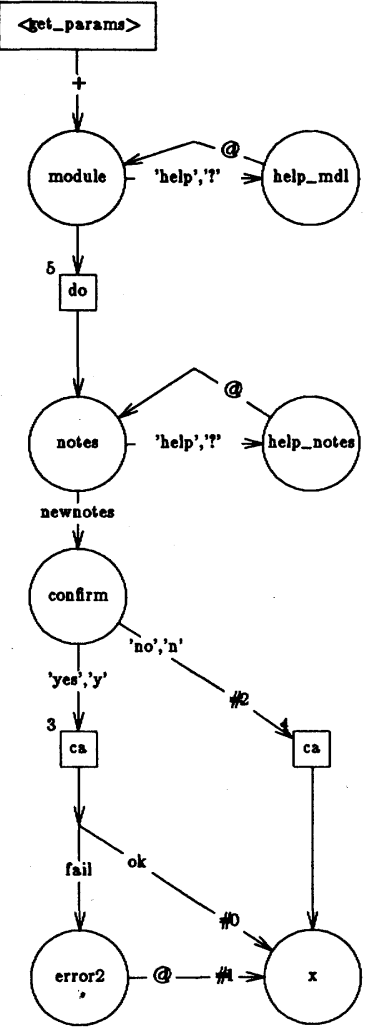

main.header,r+2;c0,c_'Add process description',mark_A

node name

tomark_A,ce,r+2,c0,'Process name:

node help_name

cs,r\$-3,c0,'Process name: string (mandatory).',

nl,'USE/DD will prevent duplicate names for any dictionary entry.',

main.lastline

node $x$

node confirm

r\$-1,c0,'Is everything OK? (y/n)'

node notes

r+2,c0,'Process notes (optional): '

node module

$r+2, c 0$,'Enter process description using text editor.'

node help_notes

cs,r6,c0,'Process notes: string (optional)'

node help_mdl

cs,r\$-3,c0,'Process module: string (optional).',

nl,'The process module is described in a file containing the process',

nl,'specifications, possibly written in Structured English.',

main.lastline

node hack1

node error 1

r\$-1,c0,'Duplicate names are not permitted.',

r\$,c0,'Type any key to continue.'

node error 2

r\$-1,c0,rv,bell, 'Unexpected database insertion error.',

r\$,c0,sv,'Type any key to continue.'

(c)

Fig. 10. (Continued.) (c) The "add_process" subconversation of data dictionary system. 


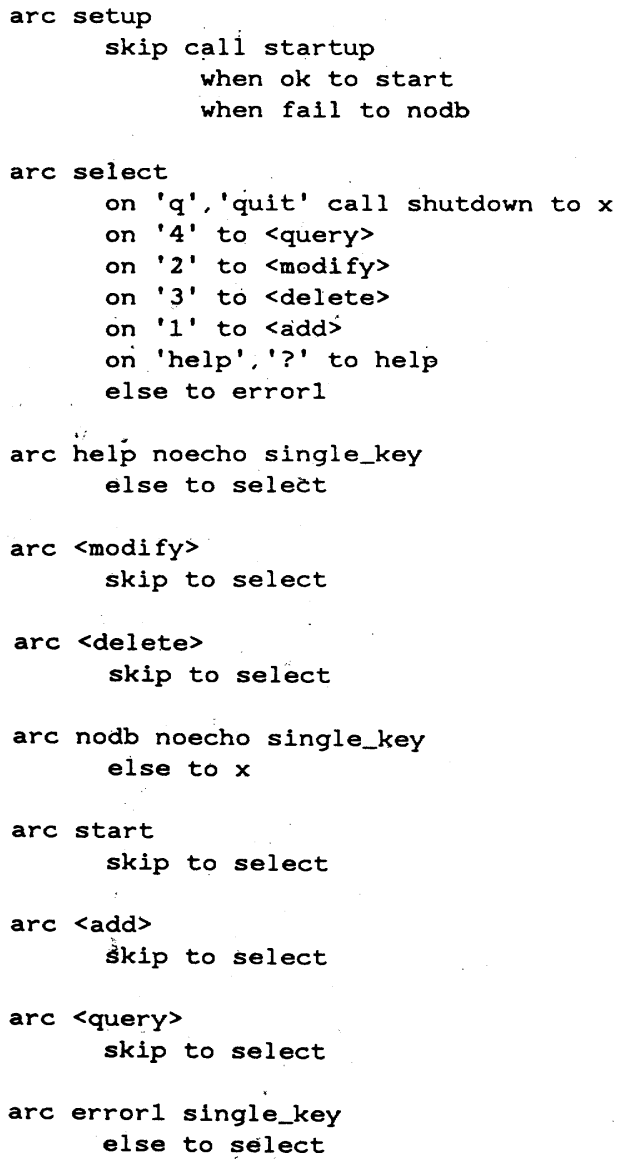

Fig. 11. Fragment of dialog description syntax from Fig. 10 (a) using RAPID/USE syntax.

tions, one is naturally drawn to determine if these ideas can be extended to such devices. Many such extensions seem feasible; in this section, we sketch out suitable strategies for these extensions. Since all of them now become minor refinements of previously discussed concepts, the treatment here is brief.

Modern bit-mapped displays are capable of displaying both textual and graphical output, of partitioning the display into two or more (possibly overlapping) regions (windows), and of either monochromatic or color display. Such displays often have a special pointing device, such as a mouse, that allows "picking" (selecting one of several buttons) and cursor movement.

One can immediately see that the notion of output specification, as associated with nodes in USE transition diagrams, may be extended to accommodate many of these concepts. For example, many pictures may be drawn by using a graphical language such as PIC [23] and simply allowing the output specification language for transition diagrams to include calls to PIC. Similarly, color definitions (or pixel values) can be associated with output nodes or with items displayed within a node, just as reverse video and standard video were used in Section III.

The problem with respect to drawing objects is not so much to draw them, but rather to be able to refer to them subsequently. Graphical interaction often involves the selection of an item, command, or icon with a pointing device. Thus, any object that might be referenced by such a device must be identifiable.

A transition diagram based strategy can only succeed if there is a way to associate placement of objects on the display with the placement of the pointing device. To achieve this, the pointing device must transmit more information than the input signal alone.

For a mouse, the "pick" buttons on top of the device may be specified and handled in the same manner as for a function button or any other single key input, as described in Section V. In other words, one could write "pick(1)" on an arc to indicate a transition from one node to another on receipt of that input. However, that transition would also require an action routine which would need information concerning the current screen position of the pointing device. This information is necessary to associate the pointing device with a displayed object, or to indicate a position at which text or additional objects should be placed.

Extension to multiple windows can also be accomplished. Windows may be edfined independently of the diagrams, and inien any transition diagram may be associated with a specific window. When a subconversation is invoked, that may cause the appearance of a new window, either at a predetermined place or at a place indicated by the location of a pointing device on the screen. This approach can work for both "persistent" windows and for "popup" windows.

Once multiple windows are introduced, then the extension to multiple concurrent dialog is also possible. This extension is handled most easily by using the location of the pointing device to determine the active dialog, but other possibilities exist as well.

In general, then, many extensions for bit-mapped graphics, multiple windows, pointing devices, and concurrency can be made within the framework of USE transition diagrams as described here. Such extensions would provide a formal basis for describing a yet larger class of interactive systems.

It is clear, though, that there are practical limits to these extensions. Even with the extensions described here, the notations would be tedious. Many sophisticated users would prefer to do "reverse specification," interactively designing the windows, icons, and user interface, and to generate the formal specification of the interaction from there.

It is important to note that transition diagrams represent a sequence of finite states, so that they become unsuitable as a representation scheme when one attempts to describe continuous input devices, such as found in many video games and drawing systems. Alternative approaches must be devised to represent continuous input.

\section{Evaluation}

In Section II, five requirements were established for a user interface notation: formalism, completeness, comprehensibility, flexibility, and testability. We conclude by evaluating USE transition diagrams against these requirements.

Formalism: Transition diagrams are a specification method for formal grammars, and have long served as a means for unambiguous specification of programming languages. The extensions made for USE transition diagrams retain that formalism, yielding an unambiguous method for dialog specification. When used in combination with formal specifications for the related actions, e.g., preconditions and postconditions, one has a formal specification methodology.

Completeness: The basic form of transition diagrams, while complete from the standpoint for specifying a formal gram- 
mar, was far from complete for specifying interactive dialogs. The extensions described here greatly extend the scope of user interaction that can be described with the diagrams, and are quite thorough for dialogs involving alphanumeric keyboards and displays, including special purpose keys and screenoriented display. We have sketched out approaches for additional extensions that are appropriate for a broader range of terminal, yet even these extensions are incomplete. We continue to search for effective descriptive techniques for broader classes of dialogs.

Comprehensibility: Transition diagrams were selected in preference to Backus-Naur form as a notation for dialog description largely on the basis of relative comprehensibility. Our own empirical evidence supports this decision. At the same time, though, additional extensions to the notation would have a negative effect upon comprehensibility. Furthermore, we have observed that understandability of the diagrams is enhanced by the use of sample screens, either using TDI or hand drawn screens, to show the typical displays from specific nodes.

Flexibility: There are three major "styles" of interactive dialogs on alphanumeric terminals: command oriented, menu selection (multiple choice), and natural language. We found that all three of these styles were easily handled with USE transition diagrams. Furthermore, we saw that we could define multiple interfaces to the same set of actions. Thus, one could build a menu selection and a command oriented interface to a system, where the menu selection might be more appropriate for the novice user, with the command approach designed for the experienced user. Similarly, one could build multilingual programs, carrying out the same set of computations in each case, but providing the user interface in two or more different languages, such as French and English.

The flexibility of the USE transition diagrams comes with a tradeoff against simplicity. Many interactive dialogs are "forms-oriented," in which the user fills in one or more entries on a screen designed to look like a form. Such systems are often specified with a "what-you-see-is-what-you-get" form layout program, as found in numerous commercially available user interface management and application generation systems. The USE transition diagram notation for such a form can be quite complex, involving a node, a transition, and possibly an action for every user input on the form.

The reason for this complexity is that the creators of the forms design system have made numerous standard decisions about the nature of the interface for their users, and hence do not need the generality provided by the USE specification method. The low-level degree of control available with the transition diagram approach is "overkill" for this class of interface, and thus requires the developer to specify in detail many features that are implicit in the form-oriented approach.

As a practical matter, we have designed and written a formsoriented program to provide a general interface to a Troll/USE relational database. It is instructive to note that this formsoriented program generates the transition diagram language used by the Transition Diagram Interpreter, from which an executable program is created. Thus, the transition diagram notation is sufficiently flexible to handle the forms-oriented approach.

Executability: The ease of machine processing of the USE transition diagrams allows informal testing of a set of alternative interfaces, so that potential system users may be presented with an executable version of an interface design and the users and dialog designers can jointly evaluate the design. The structure of RAPID/USE allows direct linkage of the executable dialog with programmed actions, so that the prototype of the dialog can be directly extended to produce a fully operational system. Thus, RAPID/USE serves the needs for prototyping and development.

Much of the work in program testing is based on coverage of program paths; these notations are easily carried over into interface testing by describing coverage of dialog paths. The formal description of the input language, as given by the USE transition diagrams, is most useful for defining a set of test cases that allow testing of the user interface. Indeed, RAPID/ USE contains logging facilities that support analysis of dialog designs, and includes the "rapsum" subsystem to generate summaries of a session with TDI.

In summary, the USE transition diagram method is a general method for the specification of human-computer interaction. It can be viewed as a pictorial programming language, encompassing many of the issues of control flow and scope of variales found in traditional programming languages. When combined with the use of the Transition Diagram Interpreter to create executable and easily modifiable versions of user interfaces, one can quickly iterate on designs of a user interface, yielding a design that is satisfactory from the user standpoint, while formalizable and implementable from the developer standpoint. The USE transition diagrams can thereby provide effective methods and tools for the engineering of interactive systems.

\section{ACKNOWLEDGMENT}

I am grateful to my colleagues who have contributed to the ideas presented in this paper. S. Stinson validated the basic ideas of using state transition diagrams for modeling interactive information systems. D. Shewmake and P. Pircher built the Transition Diagram Interpreter and the RAPID/USE system. C. Mills and P. Pircher built the Transition Diagram Editor used as a front-end to RAPID/USE. M. Kersten built the Troll/ USE relational database management system. Their help has been invaluable.

\section{REFERENCES}

[1] A. I. Wasserman, "The user software engineering methodology: An overview," in Information System Design MethodologiesA Comparative Review, A. A. Verrijn-Stuart, Ed. Amsterdam, The Netherlands: North-Holland, 1982, pp. 591-628.

[2] - "USE: A methodology for the design and development of interactive information systems," in Formal Models and Practical Tools for Information System Design, H.-J. Schneider, Ed. Amsterdam, The Netherlands: North-Holland, 1979, pp. 31-50.

[3] A. I. Wasserman and D. T. Shewmake, "Rapid prototyping of interactive information systems," $A C M$ Software Eng. Notes, vol. 7 , no. 5, pp. 171-180, Dec. 1982.

[4] A. I. Wasserman, D. D. Sherertz, M. L. Kersten, R. P. van de Riet, and M. D. Dippé, "Revised report on the programming language 
PLAIN," ACM SIGPLAN Notices, vol. 16, no. 5, pp. 59-80, May 1981.

[5] M. L. Kersten and A. I. Wasserman, "The architecture of the PLAIN data base handler," Software-Practice and Experience, vol. 11 , no. 2 , pp. $175-186$, Feb. 1981.

[6] A. I. Wasserman, "The unified support environment: Support for the user software engineering methodology," in Proc. IEEE Comput. Soc. SoftFair Conf., July 1983, pp. 145-153.

[7] A. I. Wasserman and M. L. Kersten, "A relational database environment for software development," Lab. Medical Inform. Sci., Univ. California, San Francisco, Tech. Rep. 65, 1983.

[8] A. I. Wasserman and S. K. Stinson, "A specification method for interactive information systems," Proc. IEEE Comput. Soc. Conf. Specification of Reliable Software, 1979, pp. 68-79.

[9] M. E. Conway, "Design of a separable transition-diagram compiler," Commun. ACM, vol. 6, no. 7, pp. 396-408, July 1963

[10] J. T. O'Neill, Ed., MUMPS Language Standard, ANSI Standard XII.1, Amer. Nat. Standards Inst., 1977.

[11] D. L. Parnas, "On the user of transition diagrams in the design of a user interface for an interactive computer system," in Proc. 24th Nat. ACM Conf., 1969, pp. 379-385.

[12] B. E. Casey and B. Dasarathy, "Modeling and validating the man-machine interface," Software, vol. 12 , no. 6, pp. 557-569, June 1982.

[13] R. J. K. Jacob, "Using formal specifications in the design of a human-computer interface," Commun. $A C M$, vol. 26 , no. 3 , pp. 259-264, Mar. 1983.

[14] P. Bieleski, "Flowcharting revisited (obsolete techniques vs. unexploited techniques)," in Conference Papers: 8th New Zealand Comput. Conf., 1983, pp. 123-139.

[15] D. Kieras and P. Polson, "A generalized transition network representation for interactive systems," in Proc. CHI '83 Human Factors in Comput. Syst., 1983, pp. 103-106.

[16] A. Goldberg and D. Robson, Smalltalk-80: the Language and its Implementation. Reading, MA: Addison-Wesley, 1983.

[17] W. Teitelman and L. Masinter, "The INTERLISP Programming Environment," Computer, vol. 14, no. 4, pp. 25-33, Apr. 1981.

[18] A. I. Wasserman, "User software engineering and the design of interactive systems," in Proc. 5th Int. Conf. Software Eng., 1981, pp. 387-393.

[19] T. DeMarco, Structured Analysis and System Specification. Englewood Cliffs, NJ: Prentice-Hall, 1979.

[20] M. L. Kersten and A. I. Wasserman, Troll/USE Reference Manual, Lab. Medical Inform. Sci., Univ. California, San Francisco, 1984.
[21] A. I. Wasserman and D. T. Shewmake, "The role of prototypes in the user software engineering (USE) methodology," in Advances in Human-Computer Interaction, H. R. Hartson, Ed. Norwood, NJ: Ablex, 1985 pp. 191-210.

[22] C. Mills and A. I. Wasserman, "A transition diagram editor," in Proc. 1984 Summer Usenix Meeting, June 1984, pp. 287-296.

[23] B. W. Kernighan, "PIC-A language for typesetting graphics," ACM SIGPLAN Notices, vol. 16, no. 6, pp. 92-98, June 1981; see also Proc. SIGPLAN/SIGOA Symp. Text Manipulation.

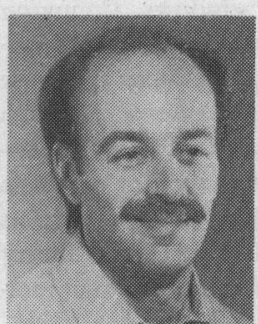

Anthony I. Wasserman (M'71) received the A.B. degree in mathematics and physics from the University of California, Berkeley, and the M.S. and Ph.D. degrees in computer sciences from the University of Wisconsin-Madison.

After three years in industry, he joined the University of California, San Francisco, where he is now Professor of Medical Information Science. Since 1970, he has also been Lecturer in the Computer Science Division at the University of California, Berkeley. He is also the founder and President of Interactive Development Environments, Inc. $\mathrm{He}$ is the architect of the User Software Engineering methodology and supporting toolset for the specification and design of interactive information systems. His research interests include software development methods, tools, and environments, human interaction with computers, and data management. He is the author of more than fifty papers and an editor of seven books, including Tutorial: Software Design Techniques (IEEE Computer Society), with P. Freeman, and Automated Tools for Information Systems Design (Amsterdam, The Netherlands: North-Holland), with H.-J. Schneider.

Dr. Wasserman is the Editor-in-Chief of ACM's Computing Surveys, and a member of the Editorial Board of several other journals, including the International Journal of Man-Machine Studies, Information Systems, and the Journal of Systems and Software. He is a member of the Programme Committee for the IFIP Congress '86, Vice-Chairman of IFIP WG 8.1 (Design and Evaluation of Information Systems), and a former chairman of ACM's SIGSOFT. He is a member of the Association for Computing Machinery and the IEEE Computer Society. 